\title{
Editorial on Special Issue: Animal models of Tourette syndrome
}

Tourette syndrome (TS) is a neurological disorder characterized by multiple repetitive movements and vocalizations called tics for a duration longer than one year (DSM-5). Although the neurobiological causes of TS have not been completely elucidated, the past decade has witnessed substantial progress in our understanding of the genetics, pathophysiology and phenomenology of the disease. This progress has brought about unique opportunities to develop novel animal models of TS, which may be instrumental in the development of novel pharmacological strategies.

The articles included in this Special Issue review these new advances and highlight emerging perspectives on the most critical problems for the validation of novel models and the translational relevance of the existing ones. The first review article discusses the application of new optogenetic and chemogenetic technologies to investigate the relevance of the cortico-striato-thalamo-cortical circuits to TS pathophysiology. Another review focuses on one of the central and most problematic issues of TS models, namely the definition and application of pathophysiological and phenomenological criteria to validate the relevance of dyskinetic movements in rodent models of tics.

New TS models in rodents are also described, derived from the chronic striatal infusion of the GABA-A receptor antagonist bicuculline (that can reproduce tic-like behaviors), and the systemic administration of the serotonergic agonist metachlorophenylpiperazine ( $\mathrm{m}$-CPP), which elicits oral movements and chewing behavior potentially mimicking abnormal motoric manifestations in TS and obsessive compulsive disorder (OCD). Although deficits in sensorimotor integration and gait are not pathognomonic in TS, they are frequently encountered in patients; thus, the first characterization of these abnormalities was performed in one of the best-validated models of TS, the D1CT-7 mouse.

Given the high comorbidity of TS with attention deficit hyperactivity disorders (ADHD), the development of refined paradigms for the measurement of attentional functions is extremely important in the characterization of animal models of TS. Thus, a new fully automated three-choice serial reaction time task designed to test cognitive functions in horses is also described, which may be particularly useful in verifying the attentional underpinnings of TS-related behaviors in this species, including their spontaneous stereotypes. Finally, the last article describes a novel 5-choice continuous performance task, a paradigm with high translational power for the assessment of attentional and cognitive deficits in TS.

Taken together, these contributions provide methodological advances for furthering our current knowledge of TS pathophysiology and thus help developing novel pharmacotherapeutic strategies for tic control and management.

We would like to thank all the contributors to this Special Issue that we hope will be an important addition to the TS literature.

\section{Marco Bortolato* Giuseppe Di Giovanni \\ * Corresponding author. E-mail address: marco.bortolato@utah.edu} (M. Bortolato)

Available online 14 October 2017 